\title{
Three Novel Autophagy-Related IncRNAs as Prognostic Biomarkers for Lung Adenocarcinoma
}

\section{Zhi-Peng Miao1, Lei Liu², Dong-Juan Wang1, Cai-Ling Jiang1, Cui-Min Zhu¹, Hua-Chuan Zheng³, Li Zhang1*}

${ }^{1}$ Department of Oncology, The Affiliated Hospital of Chengde Medical University, Chengde, China

${ }^{2}$ College of Basic Medicine, Chengde Medical University, Chengde, China

${ }^{3}$ Department of Oncology and Experimental Center, The Affiliated Hospital of Chengde Medical University, Chengde,

China

Email: sytamina@163.com, homingreceptor@hotmail.com, 461110967@qq.com, 1103470411@qq.com,

minminzhu1006@163.com,285996164@qq.com, ^cd_zhangl@126.com

How to cite this paper: Miao, Z.-P., Liu, L., Wang, D.-J., Jiang, C.-L., Zhu, C.-M., Zheng, H.-C. and Zhang, L. (2021) Three Novel Autophagy-Related lncRNAs as Prognostic Biomarkers for Lung Adenocarcinoma. Advances in Lung Cancer, 10, 65-77. https://doi.org/10.4236/alc.2021.104007

Received: November 17, 2021

Accepted: December 26, 2021

Published: December 29, 2021

Copyright $\odot 2021$ by author(s) and Scientific Research Publishing Inc. This work is licensed under the Creative Commons Attribution International License (CC BY 4.0)

http://creativecommons.org/licenses/by/4.0/

\section{Open Access}

\begin{abstract}
Background: In the present study, autophagy-related long non-coding RNAs (lncRNAs) in lung adenocarcinoma (LUAD) were screened for diagnosis and prognosis, and the molecular mechanisms of LUAD at the genetic level were investigated. Methods: From The Cancer Genome Atlas (TCGA) database, 497 gene expression data and 436 clinical data of LUAD cases were collected for analysis. In addition, 232 autophagy-related genes (ARGs) were extracted from the Human Autophagy Database (HADb). Spearman rank correlation test and the Akaike information criterion (AIC) were performed to screen the data. After filtering, a survival model including three autophagy-related lncRNAs was generated. Based on the following formula: risk score $=\Sigma$ Coef gene i $\times$ Gene i expression, the risk score of all LUAD patients could be calculated. LUAD patients were divided into two groups based on risk score for survival curve using Kaplan-Meier survival analysis. Both univariate and multivariate survival analyses were used to determine whether the three lncRNAs were independent prognostic factors using the survival package in R. Furthermore, the receiver operating characteristic (ROC) curves of clinical data were created to assess the stability of the survival model. Finally, the Gene Set Enrichment Analysis (GSEA) was used for analysis of related pathways. Results: A prognostic model consisting of three lncRNAs (AC011477.2, AC099850.3, and TRG-AS1) was generated for analysis. The 5-year survival rate in the high-risk group was $26.51 \%$ (95\% CI: $0.1842-0.382)$, which was
\end{abstract}


statistically lower than in the low-risk group 41.6\% (95\% CI: $0.307-0.563, \mathrm{P}<$ 0.05). The area under the ROC curve (AUC) of risk score was 0.700 , indicating a higher diagnostic accuracy of risk score. The results of GSEA showed enrichment in 36 pathways, including pyrimidine metabolism, pentose phosphate pathway, citric acid cycle, and cell cycle in the high-risk group, and FC-EPSILON-RI signal pathway, intestinal immune network produced by IgA, and $\mathrm{ABC}$ transporters in the low-risk group. Conclusion: The prognosis model composed of autophagy-related lncRNAs, AC011477.2, AC099850.3, and TRG-AS1, in LUAD can be used to predict the prognosis of LUAD patients and is expected to improve clinical treatment.

\section{Keywords}

Lung Adenocarcinoma, lncRNA, Prognostic Model, Autophagy, AC011477.2, AC099850.3, TRG-AS1

\section{Introduction}

Lung cancer is currently the most common and lethal malignancy worldwide. The 2-year relative survival rate for non-small cell lung cancer (NSCLC) increased from 34\% diagnostic rate between 2009 and 2010 to $42 \%$ between 2015 and 2016, including an absolute increase of $5 \%$ to $6 \%$ for each diagnostic stage [1]. Globocan 2020 data indicate the incidence and mortality of lung cancer are $37.0 \%$ worldwide and $39.8 \%$ in China [2]. Different histological subtypes have various origins, morphological appearance, epigenetic changes, clinical responses, and outcomes [3]. Therefore, investigating diagnostic and prognostic genes or markers for different subtypes could help implement precision therapies and improve patient survival.

Autophagy and apoptosis play important roles in lung cancer progression [4]. Eukaryotic cells respond to different stimuli and stress, such as starvation, hypoxia, drugs, and other harmful stimuli. To maintain cellular homeostasis, the degradation process of eukaryotic cells is regulated by autophagy-related genes (ARGs) [5]. Autophagy is a lysosomal degradation pathway physiologically involved in differentiation, survival, development and homeostasis, and pathologically in infection, cancer, neurodegeneration and aging as well as heart, liver, and kidney disease [6]. Notably, lncRNAs may act as competing endogenous RNAs (ceRNAs) which competitively bind with target miRNAs to regulate mRNAs [7]. In several studies, autophagy was shown to promote the tolerance of tumor cells to radiotherapy and chemotherapy, and lncRNAs play a crucial role in these processes [8] [9].

In the present study, the autophagy-related lncRNAs were mined from The Cancer Genome Atlas (TCGA) by searching for the lncRNA co-expressed genes of ARGs in LUAD and identified the autophagy lncRNAs associated with patient clinical data to investigate the molecular mechanisms of LUAD. 


\section{Materials and Methods}

\subsection{The Gene Expression and Clinical Data of LUAD Patients}

The gene expression and clinical data of LUAD patients were downloaded from TCGA database (https://portal.gdc.cancer.gov/). In this study, among 551 LUADs, 497 tumor samples were compared with 54 adjacent tissues, and their gene expression and clinical data were collected. In a previous study, the $M$ stage in the TNM staging system was suggested to be an unreliable independent prognostic factor [10], therefore, the missing and the $M$ stage clinical data were excluded. In addition, after filtering, 497 cases of gene expression and 436 cases of clinical data were analyzed.

\subsection{Autophagy-Related IncRNA Genes in LUAD}

A list of 232 ARGs was collected from the Human Autophagy Database (HADb) (http://www.autophagy.lu/). After Spearman rank correlation test, 311 autophagy-related lncRNAs and their coefficient values were obtained using the following criteria: correlation $>0.7$ and $\mathrm{P}<0.001$.

\subsection{The Autophagy-Related IncRNA Prognostic Model}

The autophagy-related lncRNAs were combined with survival data and then a univariate Cox's proportional hazard analysis was performed as well as Kaplan-Meier analysis using the survival package in R. A P-value $<0.05$ was considered statistically significant. To determine the optimum prognostic autophagy-related lncRNAs, the minimum Akaike information criterion (AIC) value was screened. Next, the OS curves of the optimum autophagy-related lncRNAs were drawn based on the gene expression levels. The LUAD patients were divided into high-risk and low-risk groups based on the median risk score calculated using the following formula: risk score $=\Sigma$ Coef gene $\mathrm{i} \times$ Gene i expression . Survival curves for each group were constructed based on Kaplan-Meier analysis.

\subsection{Stability Evaluation of the Prognostic Model}

To assess the stability of the constructed prognostic model, the clinical data were combined with the risk score of each LUAD patient. The clinical data included age, gender, clinical stage, $\mathrm{T}$ stage, and $\mathrm{N}$ stage. Then, univariate and multivariate prognostic analyses were performed to determine whether the three lncRNAs were independent prognostic factors using the survival package in $\mathrm{R}$. The area under the ROC curve (AUC) of risk score and different clinical data were created to assess the stability for 1-year survival time using the survival ROC package in $\mathrm{R}$.

\subsection{Gene Set Enrichment Analysis (GSEA)}

The Gene Set Enrichment Analysis (GSEA) was used to analyze the related pathways by choosing “c2.cp.kegg.v7.0.symbols.gmt" gene sets as reference gene sets. The above analysis was performed using GSEA 4.1.0 software. 


\section{Results}

\subsection{The Optimum Three Prognostic Autophagy-Related IncRNAs}

The raw data contained 19,658 mRNAs and 14,142 lncRNAs. Spearman rank correlation test, univariate Cox's proportional hazard analysis, and Kaplan-Meier analysis were used to screen 311 autophagy-related lncRNAs. Five prognostic autophagy-related lncRNAs were obtained: AC090948.1, AC011477.2, AC099850.3, TRG-AS1, and AC067852.3 (Table 1). Three optimum prognosis autophagy-related lncRNAs were acquired based on the minimum AIC value of 1551.55: AC011477.2, AC099850.3, and TRG-AS1 (Table 2).

\subsection{Construction of Prognostic Model}

The risk score of all LUAD patients was calculated based on the following formula: risk score $=\Sigma$ Coef gene $\mathrm{i} \times$ Gene i expression.

Risk score $=-0.179635915 \times$ AC011477.2 + $0.034555529 \times$ AC099850.3 $0.234455556 \times$ TRG-AS1

Based on the median risk score (cutoff value $=1.02407$ ), the LUAD patients were divided into high-risk and low-risk groups. A Sankey diagram of autophagy-related mRNA-lncRNA co-expression network was established (Table 3 and Figure 1).

To establish the optimal prognostic risk model, the survival curves of the three autophagy-related lncRNAs were drawn using survival package in $\mathrm{R}$. The overall survival (OS) of patients with high TRG-AS1 and AC011477.2 expression levels was longer (Figure 2(a) and Figure 2(b)). Similarly, patients with a high AC099850.3 expression level correlated with poor OS (Figure 2(c)). Furthermore, all LUAD patients were divided into high-risk or low-risk groups based on the median risk score of 1.02407. Then, an OS curve based on Kaplan-Meier survival analysis was performed; LUAD patients in the low-risk score group had

Table 1. Five prognostic autophagy-related lncRNAs.

\begin{tabular}{cccccc}
\hline gene & KM & HR & HR.95L & HR.95H & p value \\
\hline AC090948.1 & 0.0029 & 0.6032 & 0.4099 & 0.8878 & 0.0104 \\
AC011477.2 & 0.0000 & 0.7855 & 0.6808 & 0.9063 & 0.0009 \\
AC099850.3 & 0.0186 & 1.0429 & 1.0183 & 1.0681 & 0.0006 \\
TRG-AS1 & 0.0391 & 0.7122 & 0.5145 & 0.9859 & 0.0408 \\
AC067852.3 & 0.0285 & 0.6220 & 0.3894 & 0.9934 & 0.0468 \\
\hline
\end{tabular}

Table 2. Three selected autophagy-related lncRNAs.

\begin{tabular}{ccc}
\hline id & coef & HR \\
\hline AC011477.2 & -0.179635915 & 0.835574376 \\
AC099850.3 & 0.034555529 & 1.035159508 \\
TRG-AS1 & -0.234455556 & 0.791001389 \\
\hline
\end{tabular}


Table 3. Network of mRNA and lncRNA.

\begin{tabular}{ccc}
\hline mRNA & lncRNA & Type \\
\hline BIRC6 & AC011477.2 & coexpression \\
WDFY3 & AC011477.2 & coexpression \\
BIRC5 & AC099850.3 coexpression \\
IFNG & TRG-AS1 & coexpression \\
\hline
\end{tabular}
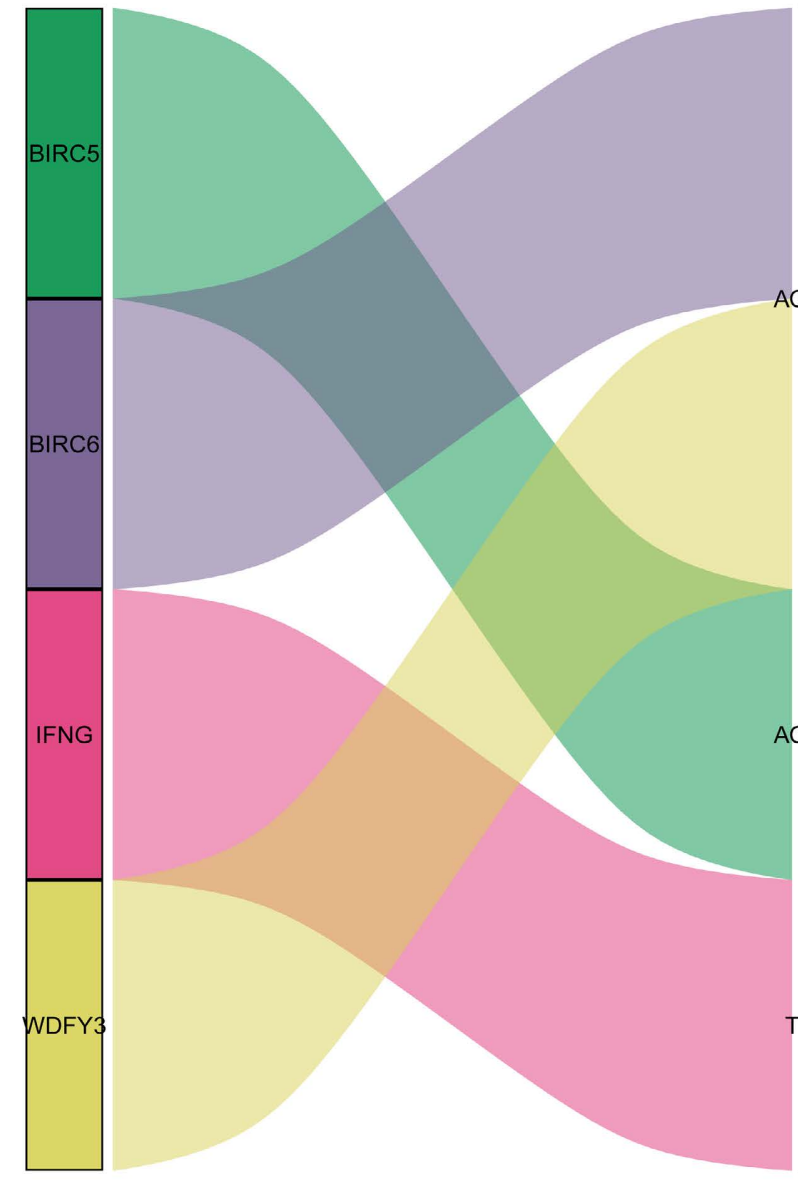

mRNA
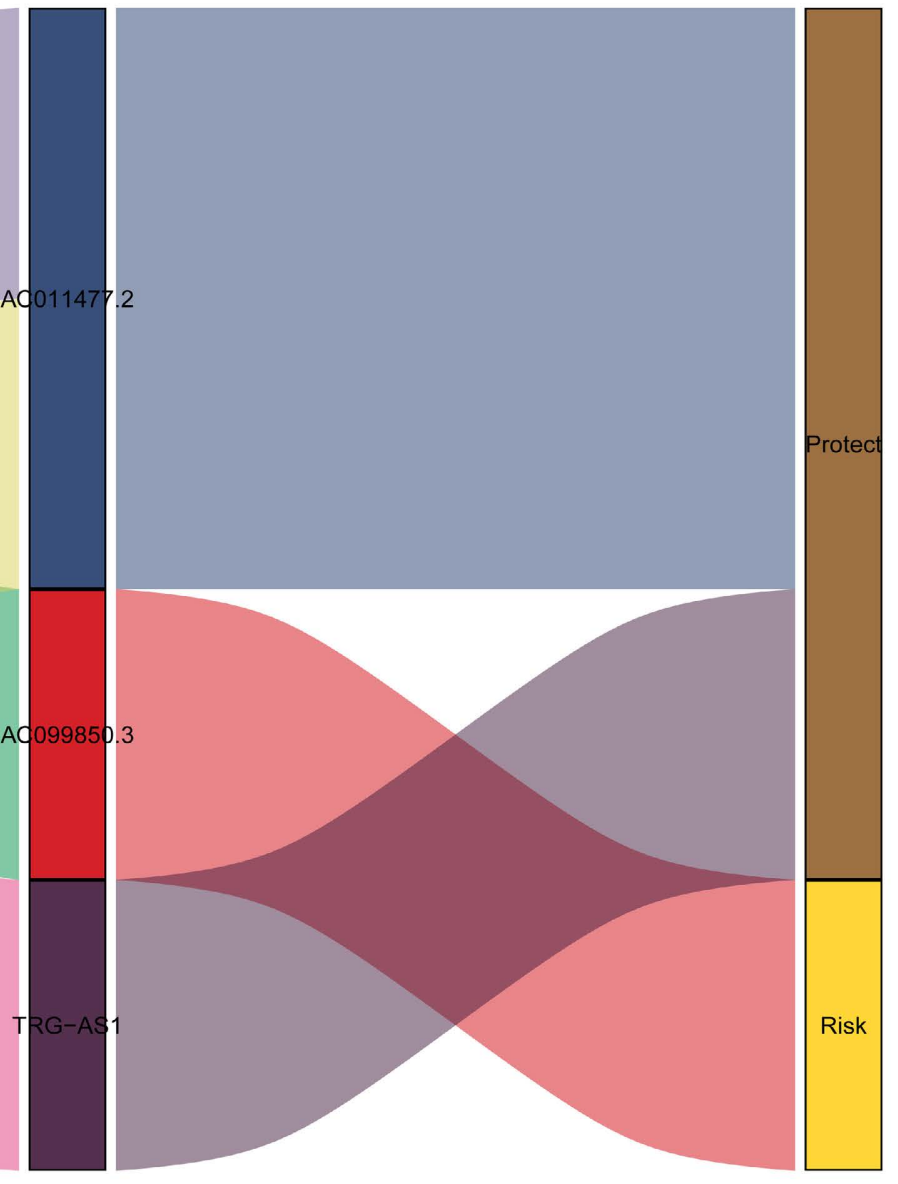

IncRNA

riskType

Figure 1. The constructed autophagy-related mRNAs-lncRNAs co-expression network was visualized by Sankey diagram.

a better prognosis (Figure 2(d), $\mathrm{P}<0.001$ ). Based on the OS curve, the 5-year survival rate in the high-risk group was $26.51 \%(95 \% \mathrm{CI}: 0.1842-0.382)$ versus 41.6\% (95\% CI: 0.307 - 0.563) in the low-risk group $(\mathrm{P}<0.05)$.

To visualize the data, the risk curve (Figure 3(a)), survival status scatter plot (Figure 3(b)), and the heat map of the three lncRNAs' expression (Figure 3(c)) were drawn.

\subsection{Stability Validation of the Prognostic Model Composed of the Three Autophagy-Related IncRNAs}

To evaluate the stability of the prognostic model composed of the three lncRNAs, 
Survival curve $(p=3.911 \mathrm{e}-02)$

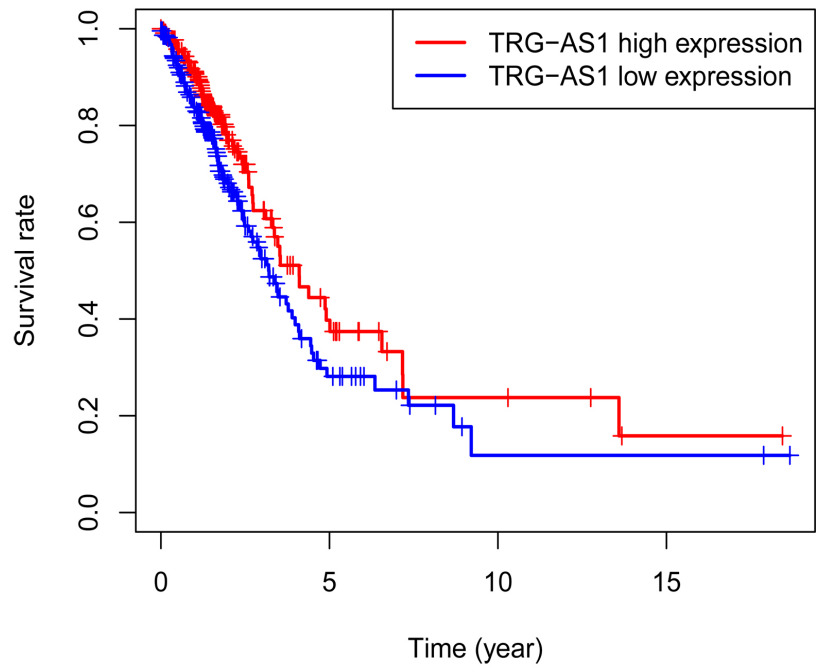

(a)

Survival curve $(p=1.858 \mathrm{e}-02)$

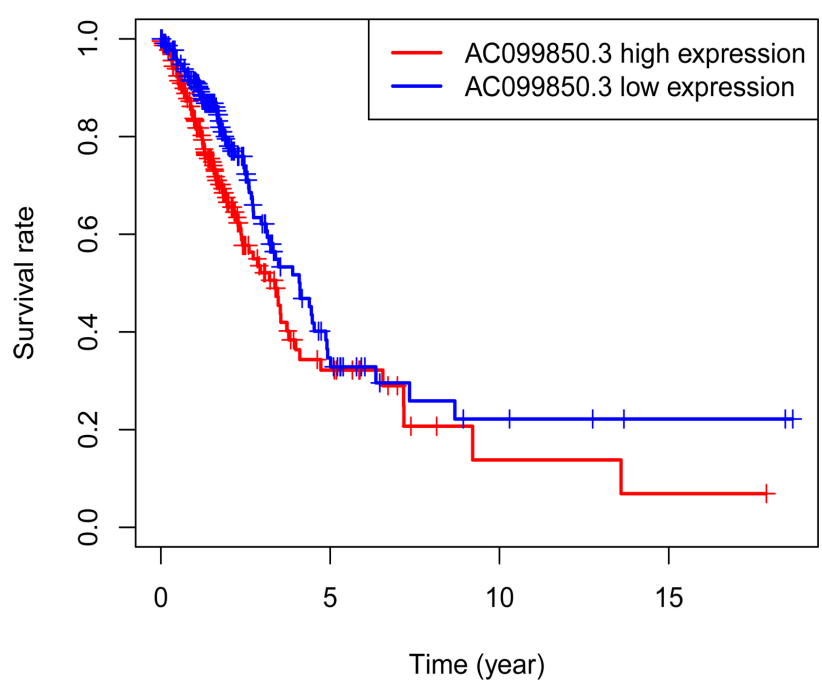

(c)
Survival curve $(p=3.092 e-05)$

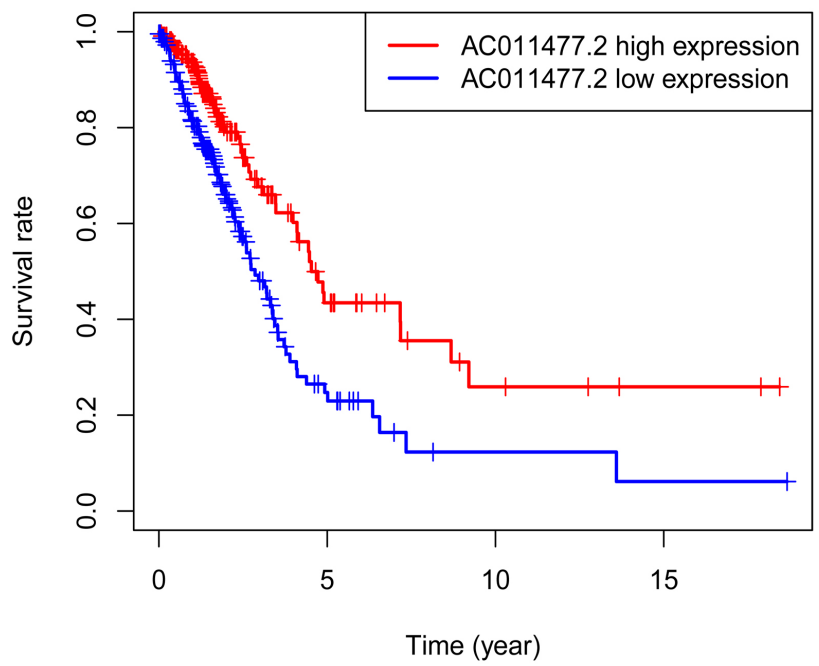

(b)

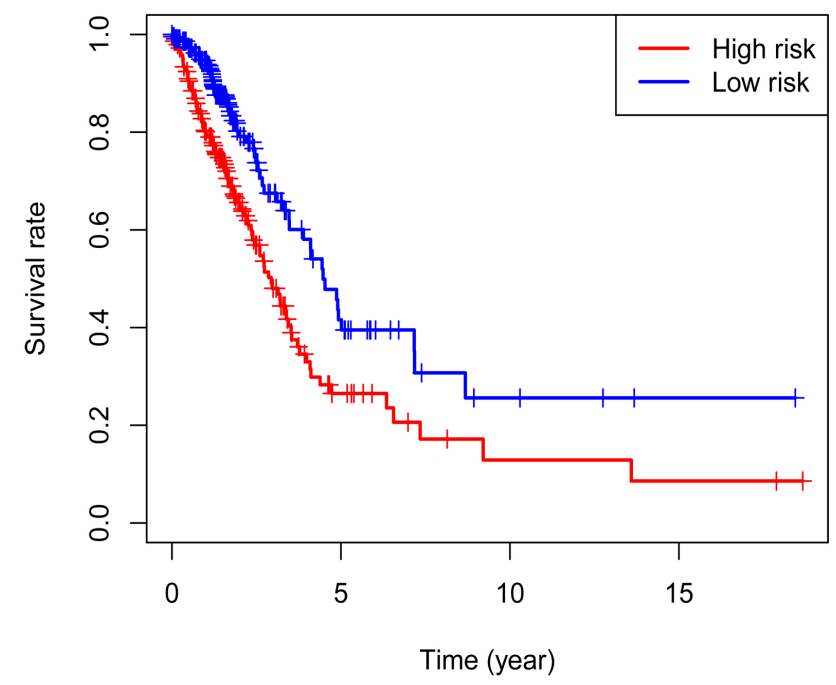

(d)

Figure 2. Survival curves of the three autophagy-related lncRNAs in LUAD. (a) TRG-AS1; (b) AC011477.2; (c) AC099850.3. (d) Kaplan-Meier survival analysis of the high-risk and low-risk groups based on the median risk score in LUAD patients.

univariate and multivariate survival analyses of 436 patients' clinical data and risk scores were performed using the survival package in $\mathrm{R}$. Based on the forest plot (Figure 4(a) and Figure 4(b)), the hazard ratio (HR) of the risk score in the univariate Cox's analysis was 2.041 (95\% CI $1.537-2.711 ; \mathrm{P}<0.001)$ and 1.972 (95\% CI $1.372-2.665 ; \mathrm{P}<0.001)$ in multivariate Cox's analysis. Therefore, the three lncRNAs (AC011477.2, AC099850.3, TRG-AS1) could be used as independent prognostic factors for LUAD patients.

To further verify the stability of this model, ROC curves were created in the prognostic model. As shown in the ROC curves (Figure 5), AUC of the risk 


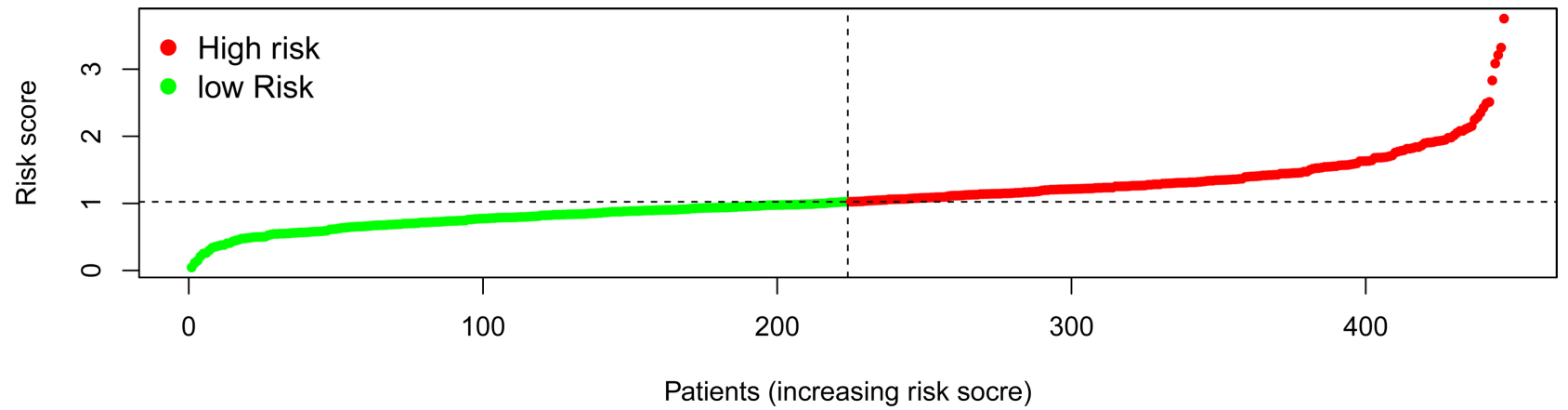

(a)

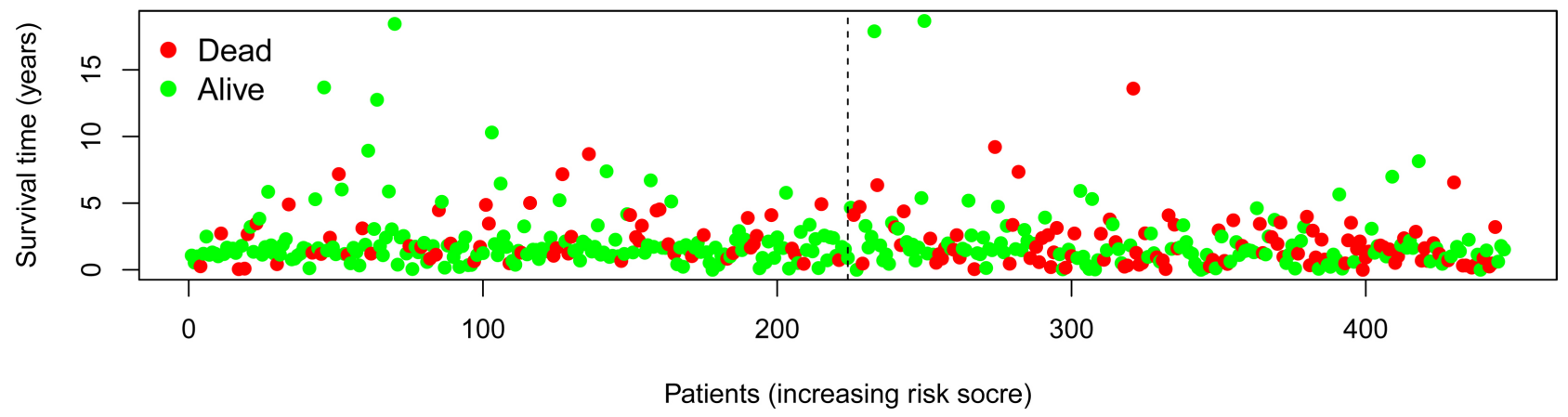

(b)

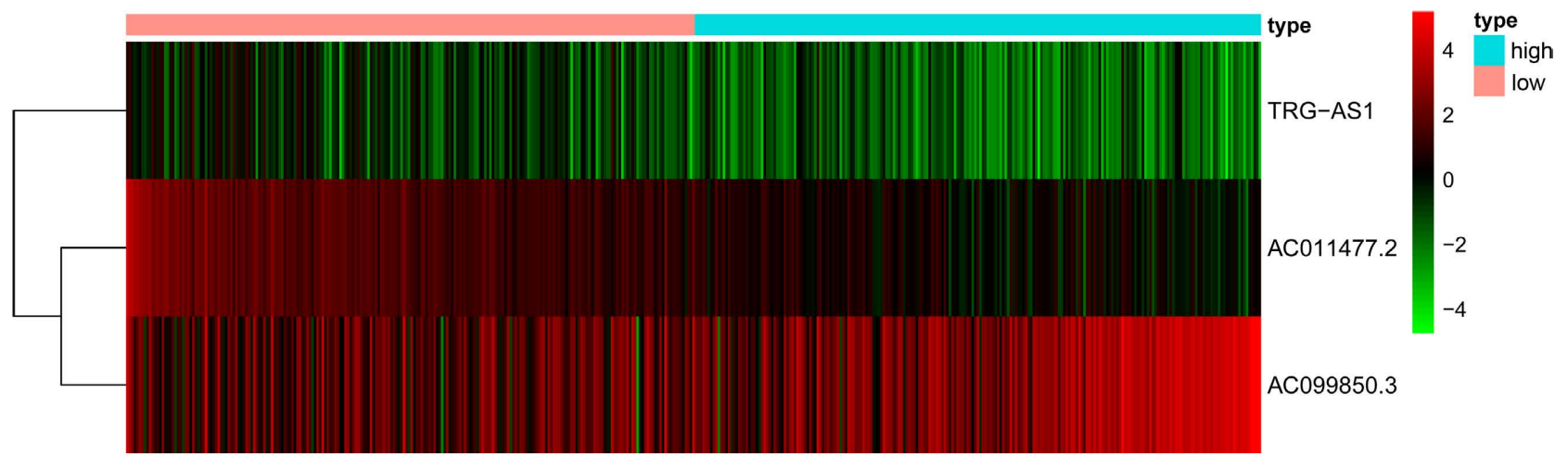

(c)

Figure 3. Autophagy-related lncRNA risk score analysis of LUAD patients. (a) the risk curve based on the risk score of each sample; (b) the scatter plot based on the survival status of each sample; (c) Heatmap showed the expression profiles of the three autophagy-related lncRNAs in the low-risk and high-risk groups.

score was 0.700 , indicating a higher diagnostic accuracy of risk score. Furthermore, the AUC of the clinical staging was 0.715 , indicating that applying clinical staging for diagnosis was equally highly valuable.

In conclusion, the stability of this prognostic model was acceptable and could be applied for the prognosis of LUAD patients.

In addition, clinical data were divided into different groups based on correlation with risk score to perform differential analysis using $t$-test (Table 4). Women had lower risk than men, the risk of clinical stage I - II was less than of stage III - IV, the risk of T1 - T2 was less than of T3 - T4, and the risk of N0 was less than of $\mathrm{N} 1-\mathrm{N} 3(\mathrm{P}<0.05)$. 


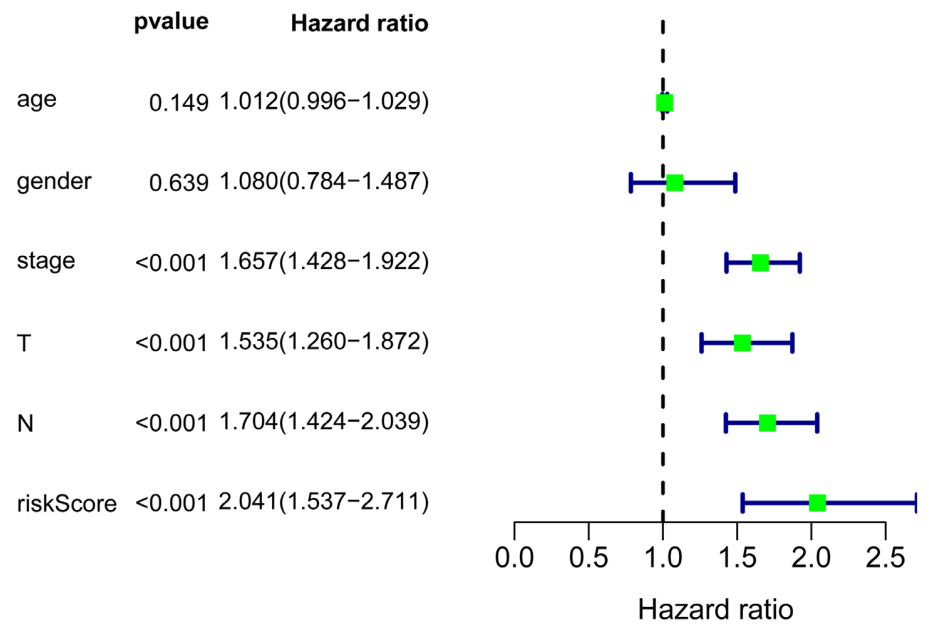

(a)

$\begin{array}{lrr} & \text { pvalue } \quad \text { Hazard ratio } \\ \text { age } & 0.022 & 1.019(1.003-1.036) \\ \text { gender } & 0.491 & 0.891(0.640-1.239) \\ \text { stage } & <0.001 & 1.439(1.160-1.784) \\ \mathrm{T} & 0.426 & 1.091(0.881-1.351) \\ \mathrm{N} & 0.131 & 1.205(0.946-1.535) \\ \text { riskScore } & <0.001 & 1.912(1.372-2.665)\end{array}$

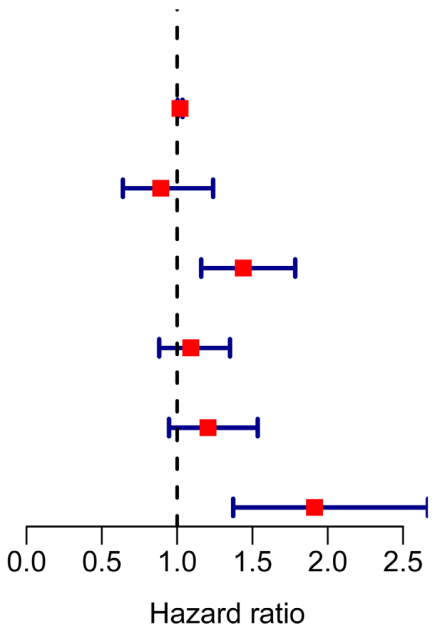

(b)

Figure 4. The univariate and multivariate Cox regression analysis of risk model score and clinical features regarding of prognostic value.

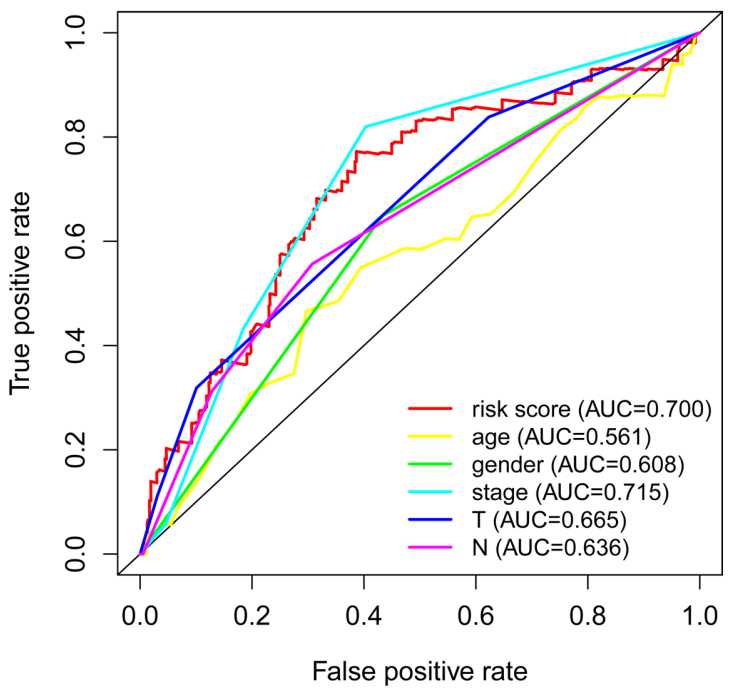

Figure 5. The ROC curves of risk score and the clinical data. 
Table 4. The association between risk score and clinical characteristics.

\begin{tabular}{ccccccc}
\hline Clinical & Group & $\mathrm{n}$ & Mean & SD & $\mathrm{t}$ & $\mathrm{P}$ \\
\hline age & $\leq 65$ & 213 & 1.125 & 0.512 & & \\
age & $>65$ & 223 & 1.074 & 0.432 & 1.1124 & 0.267 \\
gender & Female & 236 & 1.051 & 0.459 & & \\
gender & Male & 200 & 1.156 & 0.483 & & 0.02 \\
stage & Stage I - II & 343 & 1.054 & 0.456 & & \\
stage & Stage III - IV & 93 & 1.266 & 0.496 & & 0 \\
$\mathrm{~T}$ & T1-2 & 380 & 1.074 & 0.45 & & \\
$\mathrm{~T}$ & $\mathrm{~T} 3-4$ & 56 & 1.268 & 0.584 & & \\
$\mathrm{~N}$ & $\mathrm{~N} 0$ & 289 & 1.043 & 0.478 & & \\
$\mathrm{~N}$ & $\mathrm{~N} 1-3$ & 147 & 1.21 & 0.444 & & \\
\hline
\end{tabular}

\subsection{GSEA}

GSEA results showed different pathways were enriched in the high-risk and low-risk groups (Figure 6). KEGG reference gene sets were performed and results indicated enrichment in 36 pathways including pyrimidine metabolism, pentose phosphate pathway, citric acid cycle, and cell cycle in the high-risk group $(\mathrm{P}<0.05)$ and enrichment in FC-EPSILON-RI signal pathway, intestinal immune network produced by IgA, and $\mathrm{ABC}$ transporters in the low-risk group $(\mathrm{P}<0.05)$.

\section{Discussion}

Over the past few decades, researchers could investigate genomes more systematically due to application of high-throughput technologies [11]. Bioinformatics, interdisciplinary medicine, statistics, computer science, and applied mathematics, are disciplines that contributed to the development of the human genome project [12].

In several studies, autophagy has been shown to affect gene expression and even clinical treatment. Autophagy was shown to affect hepatocarcinogenesis and progression in several studies by regulating liver cancer immunity, oxidative stress, and cellular metabolism, including suppressing hepatocarcinogenesis at an early stage, promoting tumor growth at a progressive stage, and increasing chemoradiotherapy resistance of cancer cells [13] [14] [15]. Zhang et al. [16] found that lncRNA CASC2 might be useful for evaluating the expression of autophagy-related LC3-I, LC3-II, and p62. Several lncRNA-related models have been applied for the prognosis of patients with malignant tumors, such as lung, colon, liver, and breast cancers [17] [18] [19]. However, autophagy-related lncRNAs have not been extensively investigated. 


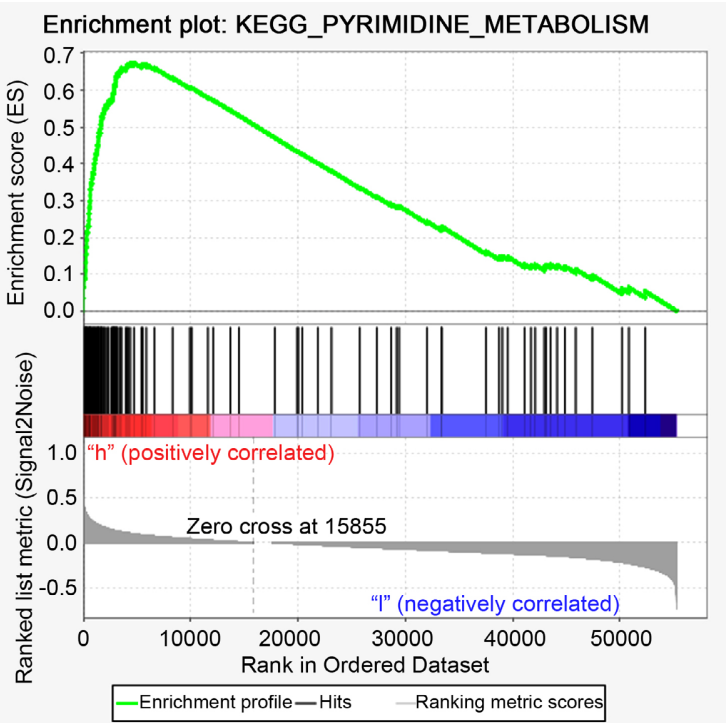

Enrichment plot: KEGG_PENTOSE_PHOSPHATE_PATHWAY

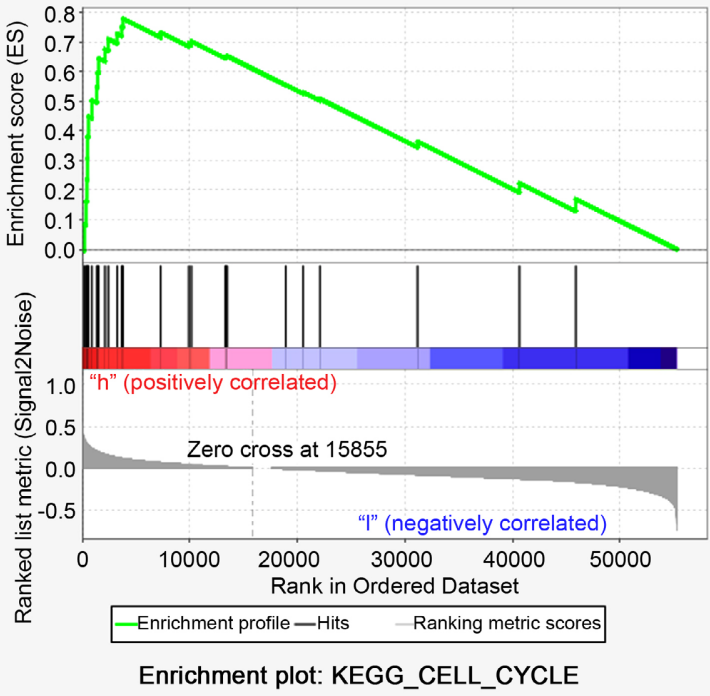

Enrichment plot: KEGG_CLTRATE_CYCLE_TCA_CYCLE
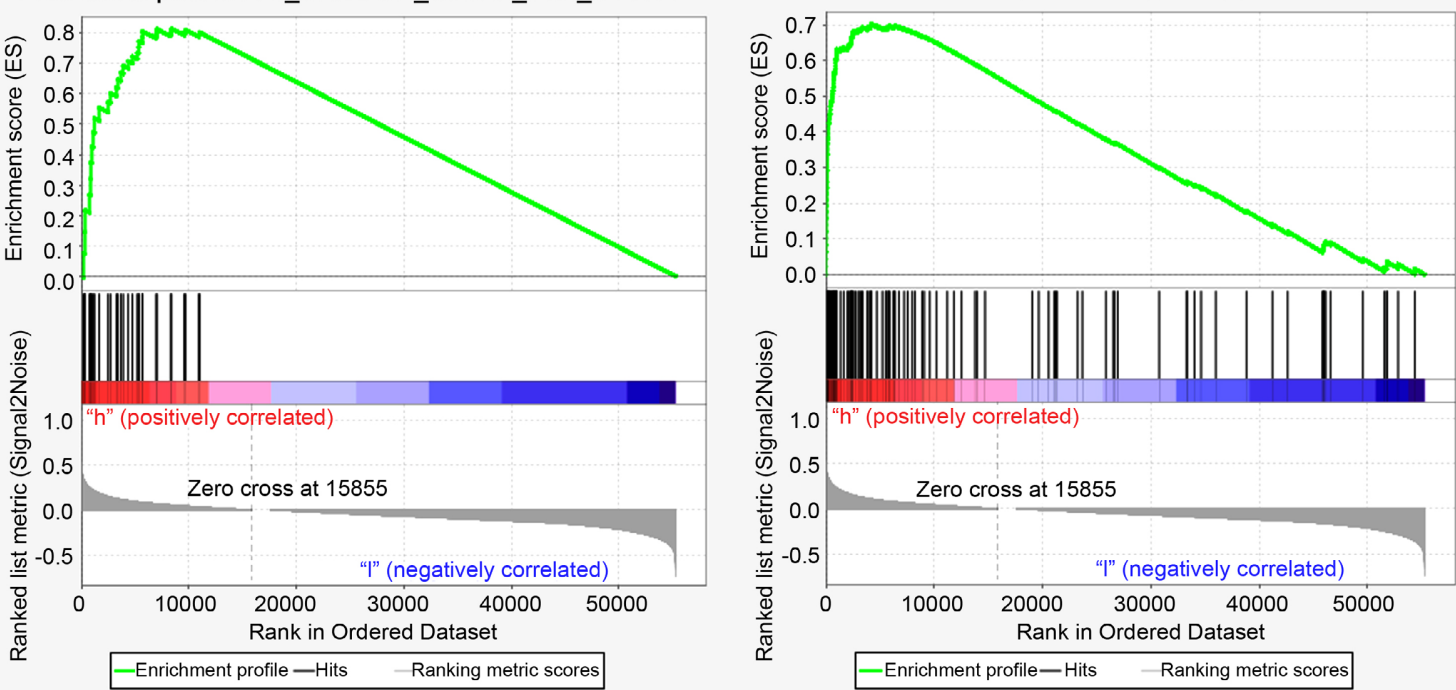

Enrichment plot

KEGG_INTESTINAL_IMMUNE_NETWORK_FOR_IGA_PRO DUCTION
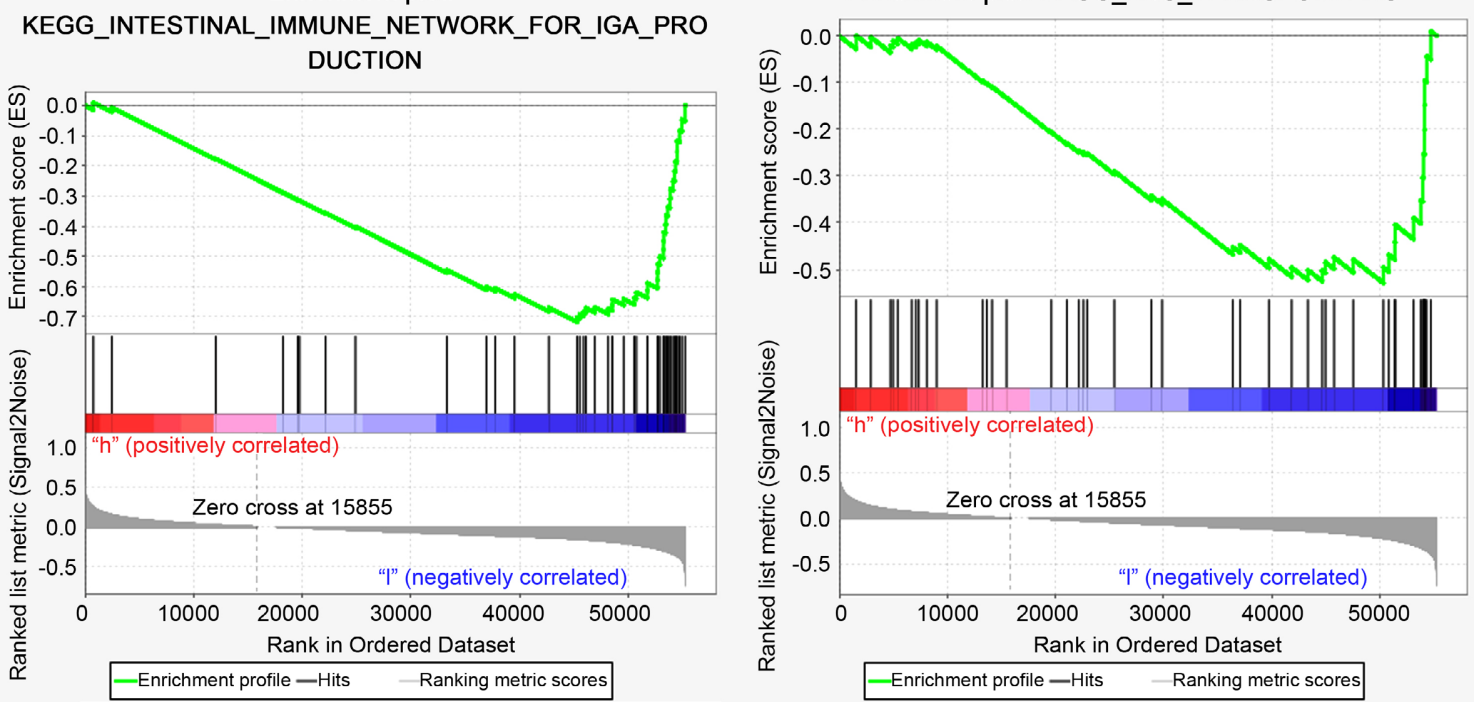

Figure 6. KEGG analysis of the three autophagy-related lncRNAs by GSEA. 
In the present study, a stable prognostic model of three autophagy-related lncRNAs (AC011477.2, AC099850.3, and TRG-AS1) in LUAD was established. AC099850.3 has been included in some hepatocellular carcinoma (HCC) prognosis studies, and the results showed that AC099850.3 may play an important role in the migration and proliferation of HCC cells. Wu et al. [20] found that AC099850.3 could promote the expression of cell cycle molecules BUB1, CDK1, PLK1, and TTK based on RT-PCR, and could inhibit CD155 and PD-L1 expression in the interference group based on Western blot analysis. The results indicated that AC099850.3 played a specific role in the immune response and warranted further research. Zhou et al. [21] showed that AC099850.3 was upregulated $(\log \mathrm{FC}>1)$ in tongue squamous cell carcinoma (TSCC).

He et al. [22] found that TRG-AS1 was highly expressed in TSCC patients and strongly associated with advanced TNM staging and poor OS. Sun et al. [23] demonstrated that TRG-AS1 was significantly overexpressed in HCC. Reportedly, TRG-AS1 silencing might inhibit the proliferation, migration, invasion, and epithelial mesenchymal transition (EMT). Xie et al. [24] found that TRG-AS1 regulated SUZ12 expression through the ceRNA of miR-877-5p, and TRG-AS1 might become a novel target for glioblastoma treatment. Notably, in the present study, TRG-AS1, a low-risk lncRNA for LUAD, had the opposite results in TSCC, liver cancer, and glioblastoma, which requires further research.

Currently, related studies in which lncRNA AC011477.2 has been investigated have not been performed. However, the present study can be used as a basis for future research on IncRNA AC011477.2 and clarify whether it has the potential to become a molecular diagnostic marker and tumor therapeutic target for LUAD.

GSEA enrichment analysis showed the high-risk group was enriched in the cell cycle, which could be associated with some of the above-mentioned effects of AC099850.3. However, further experimental proof is required. The results of the present study can be used for future prospective experiments to verify whether this prognostic model can be applicable for evaluating the prognosis of patients and identifying the specific mechanism(s) of these three lncRNAs in LUAD patients.

\section{Conclusion}

In the present study, an autophagy-related IncRNA prognostic model for LUAD consisting of AC011477.2, AC099850.3, and TRG-AS1 was established. The model showed acceptable stability for predicting the prognosis of LUAD patients and can be used for subsequent prospective experiments to improve clinical treatment.

\section{Funding Information}

Funding was provided by The National Natural Science Foundation of China (grant No. 81703001), the Natural Science Foundation of Hebei Province (grant 
No. H2020406050, H2021406021).

\section{Conflicts of Interest}

The authors declare no conflicts of interest regarding the publication of this paper.

\section{References}

[1] Siegel, R.L., Miller, K.D., Fuchs, H.E. and Jemal, A. (2021) Cancer Statistics, 2021. CA: A Cancer Journal for Clinicians, 71, 7-33. https://doi.org/10.3322/caac.21654

[2] Sung, H., Ferlay, J., Siegel, R.L., Laversanne, M., Soerjomataram, I., Jemal, A., et al. (2021) Global Cancer Statistics 2020: GLOBOCAN Estimates of Incidence and Mortality Worldwide for 36 Cancers in 185 Countries. CA: A Cancer Journal for Clinicians, 71, 209-249. https://doi.org/10.3322/caac.21660

[3] Sun, R., Liu, Z., Wang, L., Lv, W., Liu, J., Ding, C., et al. (2015) Overexpression of Stathmin Is Resistant to Paclitaxel Treatment in Patients with Non-Small Cell Lung Cancer. Tumor Biology. The Journal of the International Society for Oncodevelopmental Biology and Medicine, 36, 7195-7204. https://doi.org/10.1007/s13277-015-3361-y

[4] Liu, G., Pei, F., Yang, F., Li, L., Amin, A. D., Liu, S., et al. (2017) Role of Autophagy and Apoptosis in Non-Small-Cell Lung Cancer. International Journal of Molecular Sciences, 18, 367. https://doi.org/10.3390/ijms18020367

[5] Zhu, Y., Wang, R., Chen, W., Chen, Q. and Zhou, J. (2020) Construction of a Prognosis-Predicting Model Based on Autophagy-Related Genes for Hepatocellular Carcinoma (HCC) Patients. Aging, 12, 14582-14592. https://doi.org/10.18632/aging.103507

[6] Kopp, F. and Mendell, J.T. (2018) Functional Classification and Experimental Dissection of Long Noncoding RNAs. Cell, 172, 393-407. https://doi.org/10.1016/j.cell.2018.01.011

[7] Tian, F., Wang, J., Zhang, Z. and Yang, J. (2020) LncRNA SNHG7/miR-34a5p/SYVN1 Axis Plays a Vital Role in Proliferation, Apoptosis and Autophagy in Osteoarthritis. Biological Research, 53, 9. https://doi.org/10.1186/s40659-020-00275-6

[8] Yu, T., Guo, F., Yu, Y., Sun, T., Ma, D., Han, J., et al. (2017) Fusobacterium Nucleatum Promotes Chemoresistance to Colorectal Cancer by Modulating Autophagy. Cell, 170, 548-563.e16. https://doi.org/10.1016/j.cell.2017.07.008

[9] Zhang, W., Yuan, W., Song, J., Wang, S. and Gu, X. (2018) LncRNA CPS1-IT1 Suppresses EMT and Metastasis of Colorectal Cancer by Inhibiting Hypoxia-Induced Autophagy through Inactivation of HIF-1 $\alpha$. Biochimie, 144, 21-27. https://doi.org/10.1016/j.biochi.2017.10.002

[10] Wu, L., Wen, Z., Song, Y. and Wang, L. (2021) A Novel Autophagy-Related lncRNA Survival Model for Lung Adenocarcinoma. Journal of Cellular and Molecular Medicine, 25, 5681-5690. https://doi.org/10.1111/jcmm.16582

[11] Chen, C., Huang, H. and Wu, C.H. (2017) Protein Bioinformatics Databases and Resources. Methods in Molecular Biology (Clifton, N.J.), 1558, 3-39. https://doi.org/10.1007/978-1-4939-6783-4_1

[12] Angarica, V.E. and Del Sol, A. (2017) Bioinformatics Tools for Genome-Wide Epigenetic Research. Advances in Experimental Medicine and Biology, 978, 489-512. https://doi.org/10.1007/978-3-319-53889-1_25 
[13] Chang, C.P., Su, Y.C., Lee, P.H. and Lei, H.Y. (2013) Targeting NFKB by Autophagy to Polarize Hepatoma-Associated Macrophage Differentiation. Autophagy, 9, 619-621. https://doi.org/10.4161/auto.23546

[14] Ichimura, Y., Waguri, S., Sou, Y.S., Kageyama, S., Hasegawa, J., Ishimura, R., et al. (2013) Phosphorylation of p62 Activates the Keap1-Nrf2 Pathway during Selective Autophagy. Molecular Cell, 51, 618-631. https://doi.org/10.1016/j.molcel.2013.08.003

[15] Zhou, Y., Sun, K., Ma, Y., Yang, H., Zhang, Y., Kong, X., et al. (2014) Autophagy Inhibits Chemotherapy-Induced Apoptosis through Downregulating Bad and Bim in Hepatocellular Carcinoma Cells. Scientific Reports, 4, Article No. 5382. https://doi.org/10.1038/srep05382

[16] Zhang, P., Pan, Y., Sun, J. and Pan, G. (2021) Aberrant Expression of LncRNA CASC2 Mediated the Cell Viability, Apoptosis and Autophagy of Colon Cancer Cells by Sponging miR-19a via NF- $\kappa$ B Signaling Pathway. International Journal of Experimental Pathology, 102, 163-171. https://doi.org/10.1111/iep.12393

[17] Wang, Y., Lin, C. and Liu, Y. (2021) Molecular Mechanism of miR-34b-5p and RNA Binding Protein HuR Binding to lncRNA OIP5-AS1 in Colon Cancer Cells. Cancer Gene Therapy. https://doi.org/10.1038/s41417-021-00342-4

[18] Wang, Z. (2021) LncRNA CCAT1 Downregulation Increases the Radiosensitivity of Non-Small Cell Lung Cancer Cells. The Kaohsiung Journal of Medical Sciences, 37, 654-663. https://doi.org/10.1002/kjm2.12387

[19] Liang, Y., Song, X., Li, Y., Chen, B., Zhao, W., Wang, L., et al. (2020) LncRNA BCRT1 Promotes Breast Cancer Progression by Targeting miR-1303/PTBP3 Axis. Molecular Cancer, 19, 85. https://doi.org/10.1186/s12943-020-01206-5

[20] Wu, F., Wei, H., Liu, G. and Zhang, Y. (2021) Bioinformatics Profiling of Five Immune-Related lncRNAs for a Prognostic Model of Hepatocellular Carcinoma. Frontiers in Oncology, 11, Article ID: 667904. https://doi.org/10.3389/fonc.2021.667904

[21] Zhou, R.S., Zhang, E.X., Sun, Q.F., Ye, Z.J., Liu, J.W., Zhou, D.H., et al. (2019) Integrated Analysis of lncRNA-miRNA-mRNA ceRNA Network in Squamous Cell Carcinoma of Tongue. BMC Cancer, 19, 779.

https://doi.org/10.1186/s12885-019-5983-8

[22] He, S., Wang, X., Zhang, J., Zhou, F., Li, L. and Han, X. (2020) TRG-AS1 Is a Potent Driver of Oncogenicity of Tongue Squamous Cell Carcinoma through microRNA-543/Yes-Associated Protein 1 Axis Regulation. Cell Cycle (Georgetown, Tex.), 19, 1969-1982. https://doi.org/10.1080/15384101.2020.1786622

[23] Sun, X., Qian, Y., Wang, X., Cao, R., Zhang, J., Chen, W., et al. (2020) LncRNA TRG-AS1 Stimulates Hepatocellular Carcinoma Progression by Sponging miR-4500 to Modulate BACH1. Cancer Cell International, 20, 367. https://doi.org/10.1186/s12935-020-01440-3

[24] Xie, H., Shi, S., Chen, Q. and Chen, Z. (2019) LncRNA TRG-AS1 Promotes Glioblastoma Cell Proliferation by Competitively Binding with miR-877-5p to Regulate SUZ12 Expression. Pathology, Research and Practice, 215, Article ID: 152476. https://doi.org/10.1016/j.prp.2019.152476 